\title{
Danish Pancreatic Cancer Database
}

This article was published in the following Dove Press journal:

Clinical Epidemiology

25 October 2016

Number of times this article has been viewed

\section{Claus Fristrup' \\ Sönke Detlefsen ${ }^{2}$ \\ Carsten Palnæs Hansen ${ }^{3}$ \\ Morten Ladekarl $\left.\right|^{4,5}$ \\ 'Department of Surgical Gastroenterology, ${ }^{2}$ Department of Pathology, Odense University Hospital, Odense, ${ }^{3}$ Department of Surgical Gastroenterology, Rigshospitalet, Copenhagen, ${ }^{4}$ Department of Oncology, \\ ${ }^{5}$ Department of Gastroenterological Surgery L, Aarhus University Hospital, Aarhus C, Denmark}

Correspondence: Claus Fristrup Department of Surgical Gastroenterology, Odense University Hospital, Skovalleen 3I, 5250 Odense SV, 5000 Odense, Denmark

Tel +45 427l 7l 42

Email claus@fristrup.net
Aim of database: The Danish Pancreatic Cancer Database aims to prospectively register the epidemiology, diagnostic workup, diagnosis, treatment, and outcome of patients with pancreatic cancer in Denmark at an institutional and national level.

Study population: Since May 1, 2011, all patients with microscopically verified ductal adenocarcinoma of the pancreas have been registered in the database. As of June 30, 2014, the total number of patients registered was 2,217. All data are cross-referenced with the Danish Pathology Registry and the Danish Patient Registry to ensure the completeness of registrations.

Main variables: The main registered variables are patient demographics, performance status, diagnostic workup, histological and/or cytological diagnosis, and clinical tumor stage. The following data on treatment are registered: type of operation, date of first adjuvant, neoadjuvant, and first palliative chemo- or chemoradiation therapy, and dates for milestones in referrals, diagnostic workup, treatment decisions, and treatment. For patients undergoing resection, data on operative evaluation of tumor stage, histological diagnosis, and duration of hospital stay are registered.

Descriptive data: Death is monitored using data from the Danish Civil Registry. This registry monitors the survival status of the Danish population, and the registration is virtually complete. All data in the database are audited by all participating institutions, with respect to baseline characteristics, key indicators, and survival. The results are published annually.

Conclusion: The Danish Pancreatic Cancer Database has registered data on 2,217 patients with microscopically verified ductal adenocarcinoma of the pancreas. The data have been obtained nationwide over a period of 4 years and 2 months. The completeness of registration was $82 \%$. The observed overall 3-year survival after diagnosis was $6 \%$.

Keywords: pancreatic cancer, national database, survival

\section{Aim of database}

Pancreatic cancer (PC) is the fourth most common cause of cancer death in the USA, whereas in Europe, it is the fourth for women and fifth for men. ${ }^{1,2} \mathrm{PC}$ is the third most common cancer in the gastrointestinal tract after colorectal and esophagogastric cancer. ${ }^{2}$

The Danish Pancreatic Cancer Database (DPCD) aims to prospectively describe the epidemiology, diagnostic workup, histological or cytological diagnosis, treatment, and outcome of patients with PC in Denmark at an institutional and national level. Furthermore, DPCD collects data for continuous quality control of diagnostic workup and treatment. DPCD also provides baseline data for epidemiological research in PC. To ensure a high quality of the registered data, they are registered online by the involved clinicians. DPCD is organized as part of the Danish Pancreatic Cancer Group (DPCG), 
a multidisciplinary group established for the publication of national guidelines and to promote research in the field of PC in Denmark.

\section{Study population}

The DPCD has included patients diagnosed with pancreatic ductal adenocarcinoma in Denmark from May 1, 2011 onward. Only patients with a histological or cytological diagnosis are included. Tumors found at autopsy are not included in DPCD. The total number of patients registered as of June 30, 2014, was 2,217, corresponding to an average monthly inclusion rate of 58 patients. Patients with pancreatic neoplasms other than ductal adenocarcinoma, eg, neuroendocrine tumors, are excluded. The data in the DPCD have been cross-referenced with the Danish Pathology Registry and the Danish National Patient Registry to ensure the completeness of registrations. Lists of missing patients are distributed annually to the participating institutions in order to enter missing data. The completeness of the registry has gradually improved from $76 \%$ the first year (2011) to $83 \%$ in 2014. From 2012, the database also includes patients with adenocarcinoma of the periampullary region and duodenum. This paper presents baseline data on patients with PC only.

\section{Main variables}

Patient age at diagnosis, tissue specimen for diagnosis (ie, core needle biopsy, fine-needle aspirate, resection specimen), precise histological diagnosis, localization of the tumor, sex, and World Health Organization performance status at the time of diagnosis and/or start of treatment are registered. Also the precise modalities used for the diagnostic workup, including imaging techniques, and the pretreatment tumor-node-metastasis stage, usually determined at the multidisciplinary team conference, are registered. The Charlson Comorbidity Index can be calculated based on data from the National Patient Registry. The following treatment variables are included: type of operation, first adjuvant or neoadjuvant therapy, and first palliative chemo- or chemoradiation therapy. For patients treated by surgery, pathological staging and margin status are registered. The type of vascular resection, if any, has been registered since 2013. Baseline data of selected variables are shown in Table 1.

In order to track and monitor the clinical pathway, several key data are registered, including the following dates: referral, end of diagnostic workup, multidisciplinary team conference, patients' acceptance of treatment proposal, and first surgical and/or oncological treatment. Table 2 shows the currently registered indicators that, due to DPCG, are the measurable key indicators of quality and desired timeline of workup and treatment initiation and fulfill the criteria setup by the Danish government by law.

\section{Follow-up}

The main end point is death, and the date of recurrence is not registered directly in the database. The overall survival is shown in Figure 1. Instead, DPCD relies on data from the Danish Civil Registry to monitor the survival status of the study population, and this registration is virtually complete. The cause of death is not registered.

\section{Examples of research}

The results with respect to baseline characteristics, key indicators, and survival (Table 2) are audited by all participating institutions and published annually. The data from DPCD are readily available to researchers, and all administrative regions in Denmark, "Danske Regioner" [Danish Regions] have established standardized application forms for usage of data from all Danish National Health Databases. Data from the DPCD are currently used in ongoing epidemiological studies. Data obtained from different national registries are

Table I TNM stage, surgical treatment, and overall survival data for patients with pancreatic cancer in Denmark during a 3-year period

\begin{tabular}{ll}
\hline Baseline Data & \\
\hline Mean age at referral (years) & $69 \pm 9.8$, range: 34-97 \\
Clinical TNM stage (cTNM) & $25(1 \%)$ \\
TI & $137(6 \%)$ \\
T2 & $531(24 \%)$ \\
T3 & $494(22 \%)$ \\
T4 & $1,030(46 \%)$ \\
Tx & $235(11 \%)$ \\
N0 & $574(26 \%)$ \\
NI & $1,408(64 \%)$ \\
Nx & $925(42 \%)$ \\
M0 & $744(34 \%)$ \\
MI & $548(24 \%)$ \\
Mx & \\
Surgical treatment & $363(16 \%)$ \\
Number of resections & $43(2 \%)$ \\
Number of patients treated & \\
with palliative surgery & $97(4 \%)$ \\
Number of patients who underwent & \\
explorative surgery & \\
Survival data for all patients & $27 \%(95 \%$ Cl: $25 \%-29 \%)$ \\
Overall I-year survival & $11 \%(95 \%$ Cl: $9 \%-12 \%)$ \\
Overall 2-year survival & $6 \%(95 \%$ Cl: $5 \%-8 \%)$ \\
Overall 3-year survival & \\
\hline
\end{tabular}

Note: Data from 2,217 patients with a histological diagnosis of pancreatic cancer in Denmark between May I, 20II, and June 30, 2014.

Abbreviations: TNM, tumor-node-metastasis; $\mathrm{Cl}$, confidence interval. 
Table 2 Indicators for the surgical cases included in the Danish Pancreatic Cancer Database

\begin{tabular}{|c|c|c|c|c|c|}
\hline Indicator & Aim & Missing data (\%) & $2013-2014 *$ & $2012-2013 *$ & $20 I I-2012 *$ \\
\hline la: Diagnostic pathway completed within 14 days & $\geq 80 \%$ & 4 & $61(55-66)$ & $53(47-59)$ & $54(46-63)$ \\
\hline $\begin{array}{l}\text { Ib: Percentage of completed resections among patients } \\
\text { scheduled for resection }\end{array}$ & $\geq 80 \%$ & 0 & $84(79-89)$ & $76(69-82)$ & $80(73-85)$ \\
\hline $\begin{array}{l}\text { lla: Surgery within } 14 \text { days after patients' acceptance of } \\
\text { resection }\end{array}$ & $\geq 90 \%$ & 0 & $55(48-6 I)$ & $47(40-54)$ & $42(35-50)$ \\
\hline $\begin{array}{l}\text { Ilb: Oncological treatment initiated within } 14 \text { days after } \\
\text { patients' consent to treatment }\end{array}$ & $\geq 90 \%$ & 11 & $93(90-95)$ & $82(77-85)$ & $84(79-88)$ \\
\hline Ilc: Microscopic R0 rate for resected patients & $\geq 80 \%$ & 0 & $89(83-93)$ & $89(83-94)$ & $95(90-98)$ \\
\hline Illa: 30-day survival after resection & $\geq 95 \%$ & 0 & $98(95-99)$ & $97(93-99)$ & $98(94-100)$ \\
\hline Illb: I-year survival after R0 resection & $\geq 60 \%$ & 0 & $76(68-83)$ & $73(64-80)$ & 7I (63-79) \\
\hline
\end{tabular}

Notes: Results are based on operations performed in the year prior to analysis of the data ("analytical period"). That is, the analytical period $2013-2014$ (fourth column) is based on the resections performed from July I, 20I2, to June 30,20I3. Diagnostic pathway: DPCD registers data regarding which modalities have been used in the diagnostic pathway and the time spent on final confirmation of the diagnosis, staging, and planning of treatment. R0 resection: Resection with tumor-free resection margins. ${ }^{*}$ The data represent the percentage of patients fulfilling the indicator and the $95 \%$ confidence intervals.

Abbreviation: DPCD, Danish Pancreatic Cancer Database.

coupled using the unique Danish civil personal registration number. This coupling enables data from all registries, including DPCD, to be readily available for research studies. ${ }^{3-6}$

\section{Administrative issues and funding}

The treatment of PC in Denmark has been centralized at four surgical centers and seven oncological departments, all of which contribute with data for the DPCD. The members of both DPCD and DPCG represent surgeons, oncologists, radiologists, and pathologists from the participating institutions. All members are formally appointed by their relevant national medical specialty society. Both DPCD and DPCG receive funding from Danske Regioner. DPCD receives statistical, epidemiological, and data management support free of charge from Danske Regioner. Likewise, the running costs of the online database are funded by Danske Regioner. Most of the development and some of the registrations are based on good will from the involved clinicians.

\section{Future development}

DPCD is currently undergoing a major revision, aiming at direct transfer of data from the National Patient Registry and the Danish Pathology Registry, avoiding the need for manual registration by surgeons and oncologists. Afterward, the study population also includes patients from whom it was not possible to obtain a cytological or histological diagnosis, in many cases due to early death. These patients, with a clinical PC diagnosis only, constitute $20 \%$ of all patients with PC in Denmark and have a median overall survival as low as 1 month..$^{7-9}$ Hence, the overall survival of patients registered in DPCD is therefore expected to decrease. The four departments of pathology handling pancreatic resection specimens in Denmark have from 2015 adopted a national, standardized protocol for pancreaticoduodenectomy specimen examination, involving multicolor inking of resection margins and specimen surfaces, axial slicing, and extensive tissue sampling. ${ }^{10}$ Using this protocol, the rate of pancreaticoduodenectomy specimens with microscopic tumor-free margins ( $\mathrm{R} 0$ resections) is expected to decrease.

\section{Conclusion}

In the DPCD, data from patients with a histological diagnosis of ductal adenocarcinoma of the pancreas are included since May 1, 2011. Moreover, data from patients with a histological diagnosis of periampullary and duodenal adenocarcinoma are included since 2012. The total number of patients with PC registered as of June 30, 2014, was 2,217, and the completeness of registration was $82 \%$. DPCD supports

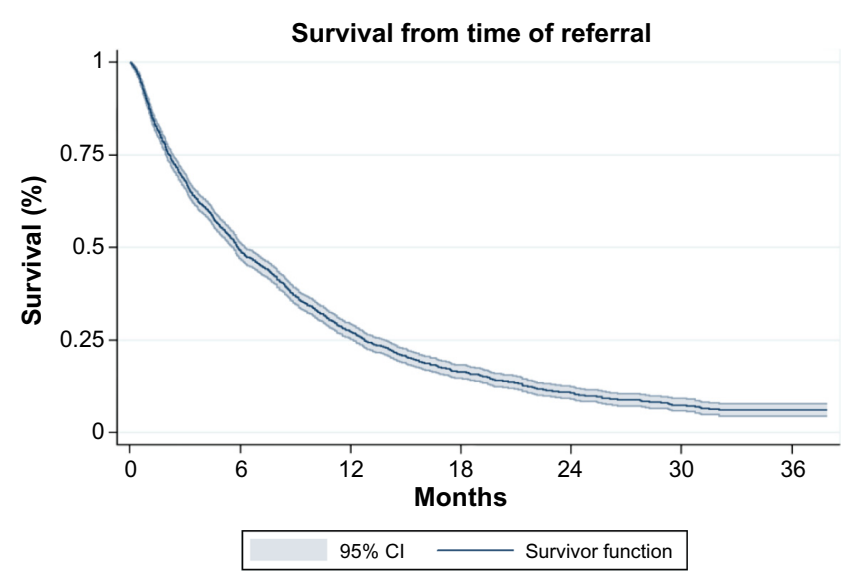

Figure I Overall survival of patients with a histological diagnosis of pancreatic cancer in Denmark during a 3-year period.

Notes: Overall survival for 2,217 patients with pancreatic cancer diagnosed between May I, 20II, and June 30, 20I4. Median survival was 5.8 months (95\% Cl: 5.6-6.3). A total of 509 (23\%) patients were still alive in September 20I4, with a median observation time of 5 months.

Abbreviation: $\mathrm{Cl}$, confidence interval. 
continuous quality control assessment of diagnostic workup, treatment, and outcome and provides baseline data for basic, epidemiological, and clinical research projects in an unselected, geographically well-defined Western population of patients with PC.

\section{Acknowledgments}

This paper was funded by the Program for Clinical Research Infrastructure (PROCRIN) established by the Lundbeck Foundation and the Novo Nordisk Foundation and administered by the Danish Regions.

\section{Disclosure}

The authors report no conflicts of interest in this work.

\section{References}

1. Siegel R, Naishadham D, Jemal A. Cancer statistics, 2013. CA Cancer J Clin. 2013;63(1):11-30.

2. Ferlay J, Steliarova-Foucher E, Lortet-Tieulent J, et al. Cancer incidence and mortality patterns in Europe: estimates for 40 countries in 2012. Eur J Cancer. 2013;49(6):1374-1403.
3. Erichsen R, Lash TL, Hamilton-Dutoit SJ, Bjerregaard B, Vyberg M, Pedersen L. Existing data sources for clinical epidemiology: the Danish National Pathology Registry and Data Bank. Clin Epidemiol. 2010;2:51-56.

4. Pedersen CB. The Danish Civil Registration System. Scand J Public Health. 2011;39(7 suppl):22-25.

5. Schmidt M, Pedersen L, Sorensen HT. The Danish Civil Registration System as a tool in epidemiology. Eur J Epidemiol. 2014; 29(8):541-549.

6. Schmidt M, Schmidt SA, Sandegaard JL, Ehrenstein V, Pedersen L, Sørensen HT. The Danish National Patient Registry: a review of content, data quality, and research potential. Clin Epidemiol. 2015;7: 449-490.

7. Teiblum S, Thygesen LC, Johansen C. Sixty-one years of pancreatic cancer in Denmark from 1943 to 2003: a nationwide study. Pancreas. 2009;38(4):374-378.

8. Bjerregaard JK, Mortensen MB, Schonnemann KR, Pfeiffer P. Characteristics, therapy and outcome in an unselected and prospectively registered cohort of pancreatic cancer patients. Eur J Cancer. 2013;49(1):98-105.

9. Nagenthiraja K, Ewertz M, Engholm G, Storm HH. Incidence and mortality of pancreatic cancer in the Nordic countries 1971-2000. Acta Oncol. 2007;46(8):1064-1069.

10. Verbeke CS, Leitch D, Menon KV, McMahon MJ, Guillou PJ, Anthoney A. Redefining the R1 resection in pancreatic cancer. $\mathrm{Br} J$ Surg. 2006;93(10):1232-1237.
Clinical Epidemiology

\section{Publish your work in this journal}

Clinical Epidemiology is an international, peer-reviewed, open access, online journal focusing on disease and drug epidemiology, identification of risk factors and screening procedures to develop optimal preventative initiatives and programs. Specific topics include: diagnosis, prognosis, treatment, screening, prevention, risk factor modification,

\section{Dovepress}

systematic reviews, risk \& safety of medical interventions, epidemiology \& biostatistical methods, and evaluation of guidelines, translational medicine, health policies \& economic evaluations. The manuscript management system is completely online and includes a very quick and fair peer-review system, which is all easy to use. 